\title{
Interplay between Anchoring and Wetting at a Nematic-Substrate Interface
}

\author{
I. Rodríguez-Ponce, ${ }^{1}$ J. M. Romero-Enrique, ${ }^{1}$ E. Velasco, ${ }^{2}$ L. Mederos, ${ }^{3}$ and L. F. Rull ${ }^{1}$ \\ ${ }^{1}$ Departamento de Física Atómica, Molecular y Nuclear, Universidad de Sevilla, Apartado 1065, Sevilla 41080, Spain \\ ${ }^{2}$ Departamento de Física Teórica de la Materia Condensada, Universidad Autónoma de Madrid, Madrid E-28049, Spain \\ ${ }^{3}$ Instituto de Ciencia de Materiales, Consejo Superior de Investigaciones Científicas, Cantoblanco, Madrid E-28049, Spain
}

(Received 30 September 1998)

\begin{abstract}
We use a generalized van der Waals molecular theory to study a model substrate-nematic interface in the regime of complete wetting and in a situation of competing interactions at the interface. The analysis shows that an anchoring transition between states with planar and homeotropic director configuration may play the role of a prewetting transition, and that reentrant anchoring must generally occur. As a result one expects complete wetting in a finite temperature range. The study provides a general context within which anchoring and wetting phenomena can be related. [S0031-9007(99)08841-9]

PACS numbers: 61.30.Cz, 64.70.Md, 68.45.Gd
\end{abstract}

An extensive research effort has been devoted in recent years to the study of the effects exhibited by liquid crystals in the presence of solid surfaces [1]. These systems are of prime importance because of both their rich phenomenology and their important applications in display technology. The tensor nature of the order parameter that describes the structure at a nematic-substrate interface gives rise, in particular, to distinct surface phenomena [2]. Of particular relevance is the phenomenon of anchoring [1] wherein the substrate induces a specific tilt angle of the nematic director at bulk with respect to the substrate normal. The associated anchoring transitions (between states of different tilt angles) have been studied experimentally [1] but there are still conceptual and experimental questions which have to find as yet a clear theoretical understanding. An intriguing problem is that posed by the experiment by Ryschenkow and Kleman [3] who observed reentrant anchoring behavior with increasing temperature: anchoring goes from being conical (tilt angle at some value between $0^{\circ}$ and $\left.90^{\circ}\right)$ to homeotropic $\left(0^{\circ}\right)$ and back to conical. An explanation of this result has been advanced in terms of the proximity to a regime of complete wetting by this isotropic phase [3,4]. However, the relation between anchoring and orientational wetting is not completely understood and available studies [1] have not provided a coherent picture in the context of which these phenomena can be explained.

In this Letter we use a molecular model to elucidate the relationship between anchoring transitions and orientational wetting, in a situation of complete wetting by the isotropic phase, when the interface undergoes the effect of competing interactions: a substrate favoring homeotropic nematic alignment and a (nascent) isotropic-nematic (IN) interface at which anchoring is planar (parallel to the interface). This setup might be relevant in most experimental situations. We show that anchoring and wetting phenomena are related in a simple manner: reentrant anchoring behavior occurs quite generally and is, in fact, a necessity in a regime of complete wetting. In turn, an an- choring transition may inhibit wetting at coexistence thus inducing a dewetting transition, with the role of the associated prewetting line played by the anchoring transition. As a result there may be a finite temperature range where the isotropic phase wets the substrate.

The theoretical model is a standard generalized van der Waals theory based on a perturbative expansion using a hard-sphere (HS) fluid as a reference system [5]. Details on the physical basis of the model and how to obtain its solutions numerically can be found elsewhere [6,7]. The relevant thermodynamic potential is the excess grand potential per unit system area $A$ over bulk, or surface tension, $\gamma[\rho]=\left(\Omega[\rho]-\Omega_{0}\right) / A$, whose functional minimum with respect to the one-particle distribution function $\rho(\mathbf{r}, \hat{\mathbf{\Omega}})$, which depends on both molecular positions $\mathbf{r}$ and orientations $\hat{\boldsymbol{\Omega}}$, gives the equilibrium structure of the interface. This function, $\rho(\mathbf{r}, \hat{\mathbf{\Omega}}) \equiv \rho(z) f(z, \hat{\mathbf{\Omega}})$, contains a mass distribution $\rho(z)$ and an angular distribution $f(z, \hat{\mathbf{\Omega}})$ which is described by a tilt angle $\psi$ giving the orientation of the nematic director with respect to the substrate normal, a nematic order parameter $\eta$ giving the amount of orientational order, and an additional biaxial order parameter. All these quantities vary locally with the distance from the substrate $z . \Omega_{0}=\Omega_{0}(\eta)$ is the bulk grand potential. The functional $\Omega[\rho]$ above is approximated in a meanfield spirit as

$$
\begin{aligned}
\Omega[\rho]= & F_{\mathrm{HS}}[\rho]+\frac{1}{2} \iiint \int d \mathbf{r} d \mathbf{r}^{\prime} d \hat{\mathbf{\Omega}} d \hat{\mathbf{\Omega}}^{\prime} \\
& \times \rho(\mathbf{r}, \hat{\mathbf{\Omega}}) \rho\left(\mathbf{r}^{\prime}, \hat{\mathbf{\Omega}}^{\prime}\right) v\left(\mathbf{r}-\mathbf{r}^{\prime}, \hat{\mathbf{\Omega}}, \hat{\mathbf{\Omega}}^{\prime}\right) \\
& -\iint d \mathbf{r} d \hat{\mathbf{\Omega}} \rho(\mathbf{r}, \hat{\mathbf{\Omega}})\left[\mu-v_{W}(\mathbf{r}, \hat{\mathbf{\Omega}})\right],
\end{aligned}
$$

where $\mu$ is the chemical potential. The isotropic hardsphere free energy $F_{\mathrm{HS}}[\rho]$ simply provides a nontrivial dependence on pressure [5], whereas the attractive potential $v$ contains the essential, anisotropic (dispersion) forces 
driving the liquid-crystalline behavior of the model material. The latter is of the form $[8,9]$,

$$
\begin{aligned}
\boldsymbol{v}\left(\mathbf{r}, \hat{\mathbf{\Omega}}, \hat{\mathbf{\Omega}}^{\prime}\right)= & v_{A}(r)+v_{B}(r) P_{2}\left(\hat{\mathbf{\Omega}} \cdot \hat{\mathbf{\Omega}}^{\prime}\right) \\
& +v_{C}(r)\left[P_{2}(\hat{\mathbf{\Omega}} \cdot \hat{\mathbf{r}})+P_{2}\left(\hat{\mathbf{\Omega}}^{\prime} \cdot \hat{\mathbf{r}}\right)\right],
\end{aligned}
$$

where $\hat{\mathbf{r}}=\mathbf{r} / r$, and $v_{A}(r), v_{B}(r)$, and $v_{C}(r)$ are functions of the intermolecular center-of-mass distance $r$ and are taken to have the simple Yukawa form $v_{i}(r)=-\epsilon_{i} \exp \left(-\lambda_{i} r\right) / r$ for $r>\sigma$, and $v_{i}(r)=0$ otherwise, where $\sigma$ is the diameter of a hard sphere.

The surface potential is modeled as $v_{W}(z, \theta)=$ $-\epsilon_{W} \exp \left[-\lambda_{W}(z-1)\right] P_{2}(\cos \theta)$, with $P_{2}$ a Legendre polynomial and $\cos \theta=\hat{\mathbf{\Omega}} \cdot \hat{\mathbf{z}}$. Numerical values for the parameters are $\epsilon_{A}=1$, which sets the temperature scale, and $\epsilon_{B} / \epsilon_{A}=0.847$, which may be typical of real nematogens [5]. In our calculations $\epsilon_{C}$ and $\epsilon_{W}$ give rise to competing effects: $\epsilon_{C}$ is positive which favors parallel director orientation at the interface, whereas $\epsilon_{W}$ is also positive favoring (strong) homeotropic anchoring at the substrate; otherwise we let these two parameters take free values. The range parameters $\lambda_{i}$ are set in units of $\sigma=1$ (throughout we choose this unit to set the length scale) as $\lambda_{i}=2,4,1.75 ; i=A, B, C$, respectively, and $\lambda_{W}=1$. We later comment on the importance of this latter parameter on the results.

The model predicts a bulk phase diagram with vapor, isotropic liquid, and nematic liquid coexisting at a triple point temperature $T_{\mathrm{NIV}}$. The surface phase diagram $\epsilon_{C^{-}} \epsilon_{W}$ corresponding to a nematic at coexistence with its isotropic phase summarizes many of the important results and is depicted in Fig. 1. A first-order transition

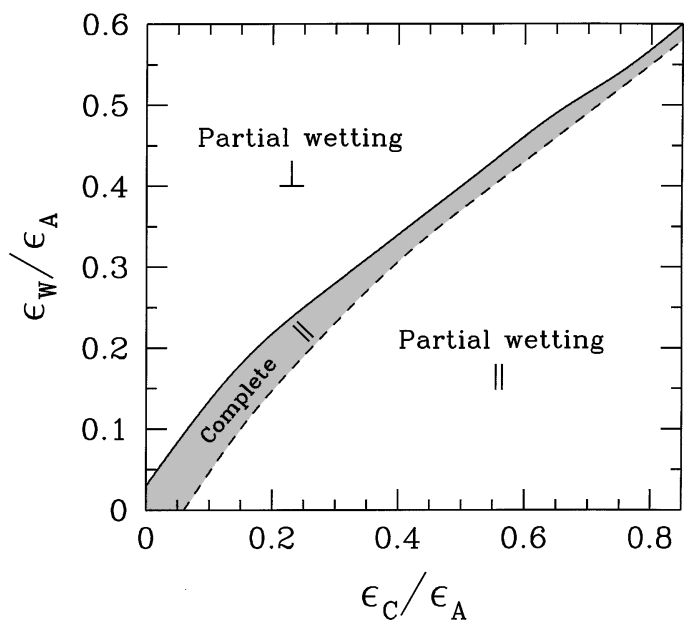

FIG. 1. Surface phase diagram in the plane $\epsilon_{C}-\epsilon_{W}$. Conditions at bulk correspond to coexistence between isotropic and nematic phases at a reduced temperature $T=0.57$. Shaded area depicts configurations of complete wetting. Symbol II denotes configurations where the director is parallel to the substrate (tilt angle $\psi=90^{\circ}$ ), symbol $\perp$ refers to $\psi=0^{\circ}$. Continuous line corresponds to the first-order anchoring transitions, whereas the dashed lines are second-order wetting transitions. (continuous curve) separates states with homeotropic $\left(\psi=0^{\circ}\right)$ anchoring from configurations with planar $(\psi=$ $90^{\circ}$ ) anchoring. [The model does not limit the order of the transition to be first order and inclusion of additional, less symmetric terms in the potential given by Eq. (2) would allow for continuous transitions.] In addition, a wetting line of second-order [10] transitions divides regions of complete wetting and regions corresponding to configurations where the isotropic phase partially wets the substrate. Between these two lines a wetting layer of isotropic material intrudes between the substrate and the nematic (shaded area) in planar configuration. The positive slope of this line can be explained by the competing effects of $\epsilon_{C}$ and $\epsilon_{W}$.

Let us focus on the anchoring transition. In our model the transition is first order and proceeds between states with homeotropic and planar orientation. Curves of surface tension as a function of the tilt angle (Fig. 2) show two relative minima located at $\psi=0^{\circ}$ and $\psi=$ $90^{\circ}$. No further minima exist and there is always a finite energy barrier between minima indicating first-order behavior. Along the anchoring transition line a thin film of isotropic material, with homeotropically oriented nematic bulk, coexists with a thick film associated with a planar tilt angle. This is evident from Fig. 3 which shows the absorption as a function of temperature, the former being defined as $\Gamma=\int_{0}^{\infty} d z\left|\eta(z)-\eta_{\infty}\right|$, where $\eta(z)$ is the nematic order parameter in the reference frame of the director, and $\eta_{\infty}$ is the bulk nematic order parameter [11]. Now an interesting question is how the anchoring transition line approaches coexistence and actually meets the coexistence line. The figure shows that the thick isotropic film becomes macroscopically thick at coexistence which indicates that the anchoring transition

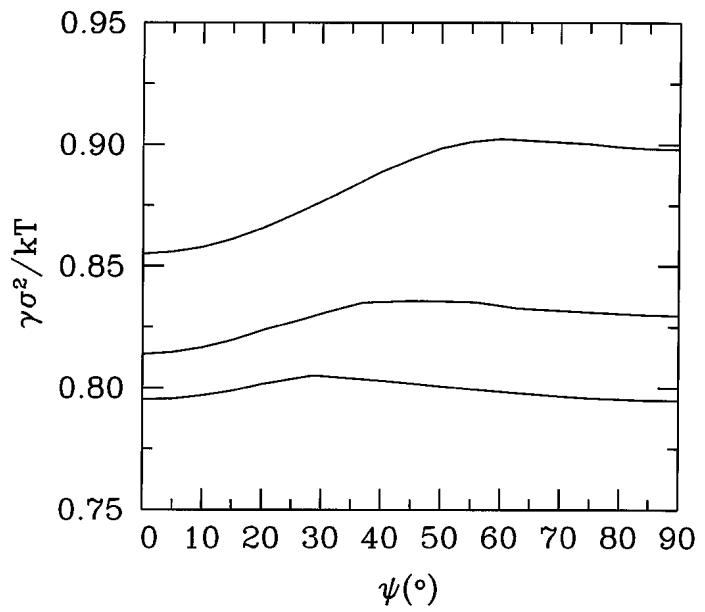

FIG. 2. Surface tension as a function of the tilt angle calculated along the nematic branch of the nematic-vapor transition, for $\epsilon_{W}=0.7$ and $\epsilon_{C}=0.807$. Values of the reduced temperature $T$ are, from top to bottom, 0.5450, 0.5500, and 0.5522; the anchoring transition occurs at the triple point $T_{\text {NIV }}=0.5522$ (bottom curve), where the two minima of equal height are separated by a finite free energy barrier. 


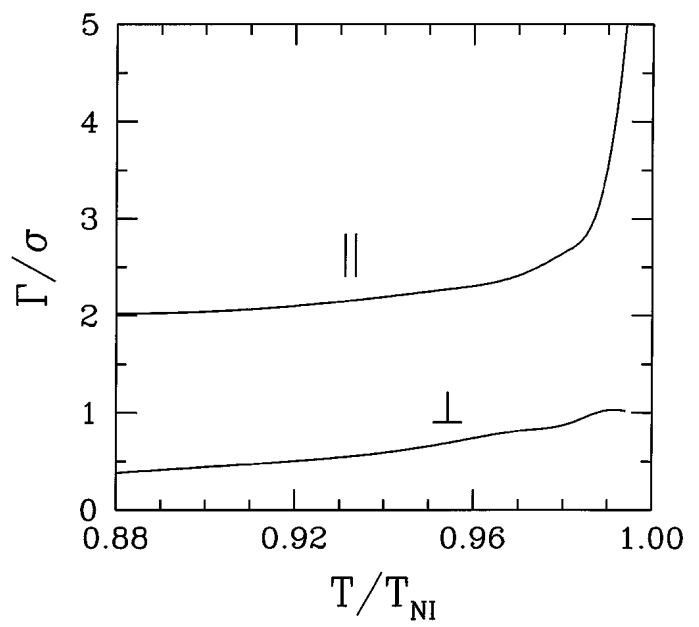

FIG. 3. Adsorption of the system as a function of temperature along the anchoring line with $\epsilon_{C}=0.807$ and $\epsilon_{W}=0.52$. The temperature has been reduced by the value of the isotropicnematic transition temperature $T_{\mathrm{NI}}$ where the anchoring line meets the coexistence line.

is associated with some kind of wetting behavior, as will be clear shortly.

Figure 4, which is part of the whole $T$ - $\rho$ plane, provides additional clues. Surface phase transitions have been superimposed on the bulk phase diagram. Anchoring transition lines for different values of $\epsilon_{W}$ are plotted. In all cases there is complete wetting by the isotropic phase in a close neighborhood above a wetting temperature $T_{W}$; the wetting transition is of second order. Sufficiently far away from coexistence the anchoring line always has a negative slope [12], $(d T / d \rho)_{A}<0$, but the lines bend upward on approaching coexistence, their slopes eventually becoming positive, $(d T / d \rho)_{A}>0$. The anchoring line terminates

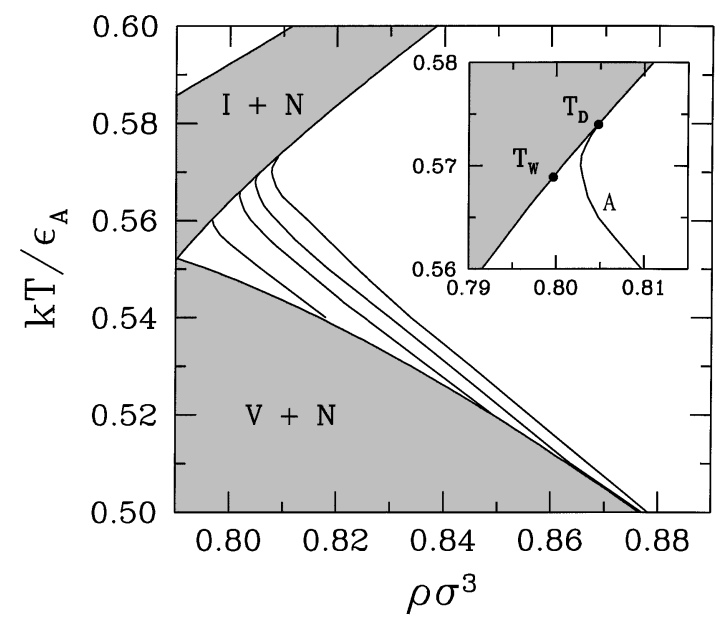

FIG. 4. Temperature vs density phase diagram in the neighborhood of the triple point $T_{\mathrm{NIV}}$. Bulk phase boundaries are indicated by thick lines, and two-phase regions have been shaded. Thin lines refer to anchoring lines for a variety of values of the surface strength $\epsilon_{W}$, from 0.52 (upper curve) through 0.54 , 0.56 , to 0.60 (bottom curve). The inset shows the topology of the phase diagram for the lowest value of $\epsilon_{W}$. on the nematic coexistence line at $T_{D}$ (see inset of the figure for an enlarged depiction); this point is a firstorder dewetting transition where a macroscopically thick film with planar director orientation at the nematic side coexists with a thin film with homeotropic orientation. The anchoring line thus plays the role of a prewetting line associated with a bulk (de-)wetting transition. Note that this prewetting line does not terminate away from bulk coexistence at a surface critical point, as is normally the case [13], since it separates phases with different symmetry. Details of one of the cases are depicted in the inset showing $T_{W}$, the wetting temperatures, $T_{D}$, the dewetting temperature, and $A$, the anchoring line. Wetting thus occurs in a finite temperature range between $T_{W}$ and $T_{D}$. A crucial observation is that thermodynamic continuity implies that the anchoring line has to meet the coexistence line tangentially and, in view of the positive slope of the latter, reentrant anchoring then follows as a necessary consequence [14].

The Ryschenkow-Kleman experiment can be interpreted in the light of the picture obtained with our molecular model. It would seem that the experimental conditions are such as to fix the density within the reentrant interval. As is apparent from Fig. 4, the temperature range where reentrant behavior can be observed is rather sensitive with respect to the surface strength, this range increasing as the surface strength decreases. This would imply that the experimental behavior could depend drastically on the type of surface treatment. The reentrant density interval is otherwise small; for example, for the weakest surface that we have considered (see inset of Fig. 4) this interval amounts to only $4 \%$ of the nematic density at the triple point. The temperature range where the reentrant planar configuration sets in is also small, $\Delta T \sim 0.4 \% T_{\mathrm{NIV}}$. Taking $T_{\text {NIV }} \sim O\left(10^{2}\right) \mathrm{K}$ (which is typical of crystalline materials) gives $\Delta T \sim O(1) \mathrm{K}$. This temperature range should be accessible experimentally.

Finally, as shown in Fig. 5, the surface strength also has an influence on the wetting and dewetting temperatures and, what is especially important, on the complete wetting temperature range. As anchoring becomes stronger $\left(\epsilon_{W}\right.$ increasing) both temperatures shift to lower values and complete wetting is restricted to a narrower temperature range. Complete wetting eventually disappears at some value of $\epsilon_{W}$ which we estimate to be $\sim 0.60$ and at a temperature still above $T_{\mathrm{NIV}}$. Anchoring transitions do survive even after complete wetting is no longer possible, but the stability region corresponding to the planar configuration shrinks quite substantially so that at $\epsilon_{W}=0.70$ there exists no anchoring transition at the triple point.

An even richer scenario is expected in the case of a first-order wetting transition. We would then have a genuine prewetting line extending off the coexistence line into the nematic region and terminating at a surface critical point [13]. This is in contrast with the behavior observed for the prewetting line associated with the 


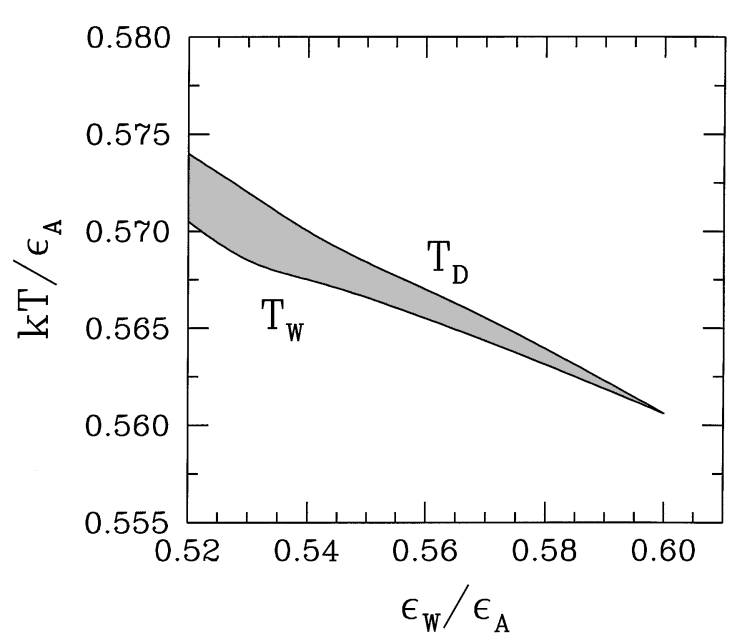

FIG. 5. Wetting $T_{W}$ and dewetting $T_{D}$ temperatures as a function of $\epsilon_{W}$ with $\epsilon_{C}=0.75$.

dewetting transition. In our model a first-order wetting transition can be induced, we believe, by changing the value of the range of the surface potential, $\lambda_{W}$, relative to the range of the fluid attractive potential. It must be noted, however, that it is crucial for an order-parameter depletion at the substrate (which is necessary for the effects reported in this study to exist) to occur that the range of the surface potential be less than some of the fluid interaction potentials. Another interesting issue, not central to this work, is how the anchoring transition behaves at low $T$. The most likely scenario is that in which the anchoring line meets a nematic spinodal (with the vapor phase or, what is more likely, with the smectic phase in case the latter is separated from the nematic by a first-order transition) or a continuous transition line with the smectic. Since our model cannot describe smectic phases we have not explored this behavior, which is left for future work.

A possible improvement of the present model involves a more accurate treatment of the hard core, which is presently assumed to be spherical. This is only an approximation since the IN transition is mostly governed by short-range hard interactions. A more sophisticated theory would probably predict an isotropic-nematic transition line with a larger slope, which would in turn decrease to some extent the temperature range of reentrant behavior. However, this region should exist out of necessity.

In conclusion, we have discussed a theoretical framework where orientational wetting and anchoring transitions can be related. We have seen that reentrant anchoring is generally expected in interfaces with competing interactions in the regime of complete wetting and this implies complete wetting in a finite temperature range because of the appearance of an upper dewetting point associated with the anchoring line. Since the conditions set up in our system (i.e., competing surface interactions) are rather general we would expect that a large variety of materials adsorbed on substrates under strong anchoring conditions might exhibit similar behavior.

This work was partly supported by Grants No. PB-940005-C02 and No. PB-94-1442 from the Dirección General de Investigación Científica y Tecnológica (DGICyT) of Spain, and ERB 4050PL930367 and CI1*94-0132 from the European Union.

[1] B. Jerome, Rep. Prog. Phys. 54, 391 (1991), and references therein.

[2] T. J. Sluckin and A. Poniewiersky, in Fluid Interfacial Phenomena, edited by C. A. Croxton (Wiley, New York, 1986), Chap. 5.

[3] G. Ryschenkow and J. S. Kleman, J. Chem. Phys. 64, 404 (1976).

[4] P. I. C. Teixeira, T. J. Sluckin, and D. E. Sullivan, Liq. Cryst. 14, 1243 (1993).

[5] M. M. Telo da Gama, Mol. Phys. 52, 585 (1984); 52, 611 (1984).

[6] F. N. Braun, T. J. Sluckin, E. Velasco, and L. Mederos, Phys. Rev. E 53, 706 (1996). An alternative numerical approach is that used in E. Martín del Río, M. M. Telo da Gama, E. de Miguel, and L. Rull, Phys. Rev. E 52, 5028 (1995).

[7] Y. Martínez, E. Velasco, A. M. Somoza, L. Mederos, and T. J. Sluckin, J. Chem. Phys. 108, 2583 (1998).

[8] J.H. Thurtell, M. M. Telo da Gama, and K. E. Gubbins, Mol. Phys. 54, 321 (1985).

[9] B. Tjipto-Margo, A.K. Sen, L. Mederos, and D.E. Sullivan, Mol. Phys. 67, 601 (1989).

[10] The wetting transition is found to be second order within the accuracy of our calculations. However, we cannot rule out that it be a very weak first-order transition.

[11] Note that this definition encompasses both planar and homeotropic interfacial structures, so that there is no ambiguity in the definition of a proper order parameter for this particular transition.

[12] This is because, as the density decreases in the planar state, surface order is weakened and the effect of the wall is reduced which reinforces the planar state.

[13] R. Pandit, M. Schick, and M. Wortis, Phys. Rev. B 26, 5112 (1982).

[14] In our model reentrant anchoring at constant pressure is not observed. Whether or not this occurs depends on the relative slope of the isobar in the $T$ - $\rho$ plane $(d T / d \rho)_{P}<0$ and that of the anchoring transition line $(d T / d \rho)_{A}$. For our model liquid crystal we always have $(d T / d \rho)_{P}>(d T / d \rho)_{A}$ when the latter is negative and isobars never cross the anchoring line more than once. 